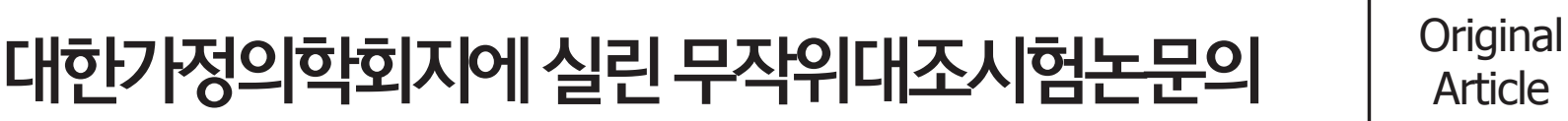 질 평가
}

정우빈, 이경우, 황인홍, 이동희, 김수영*

한림대학교 의과대학 강동성심병원 가정의학과

\section{Quality Assessment of Randomized Controlled Trials in the Journal of the Korean Academy of Family Medicine}

\author{
Woobin Chung, Kyung Woo Lee, In Hong Hwang, Dong Hee Lee, Soo Young Kim* \\ Department of Family Medicine, Hallym University College of Medicine, Seoul, Korea
}

Background: Because low quality trials may lead to wrong conclusions, quality assessments are necessary. Thus, this study scrutinizes randomized controlled trials (RCTs) in the Journal of the Korean Academy of Family Medicine (KAFM) to assess the quantity and the quality.

Methods: Upon extracting randomized controlled trials from all the articles published in the Journal of the KAFM from 1980 to 2005, assessments were made on the Jadad scale and the adequacy of allocation concealment. The selections and assessments were performed independently by two researchers, and adjustment of the differences were done by a thirdparty researcher. In addition, the factors that may affect the quality levels were analyzed.

Results: Twenty-three trials were included. In 1980s, there were only $1.09 \%$ of the total original articles, but in 2000 s, $2.63 \%$ were RCTs. The mean total Jadad score increased from 1 point in 1980s to 2.17 in 2000s, and the "good quality" trials also increased from $0 \%$ to $33.33 \%$. As for the adequate allocation concealment, however, only two studies were observed in 2000s. The most lacking aspect was the appropriate double-blinding. Also, studies intervened by medicine or funded or examined by Institutional Review Boards (IRBs) tended to receive higher quality assessments.

Conclusion: Although RCTs consistently increased in quantity and quality, in future studies, researchers should continue to strive towards achieving adequate allocation concealment and appropriate double-blinding. In addition, researchers must become more interested in receiving funds and examination by IRBs.

Keywords: Journal of the Korean Academy of Family Medicine; Randomized Controlled Trial; Quality Assessment; Allocation Concealment

\footnotetext{
서론

관찰 연구가 주가 되던 이전에 비해 근래는 치료에 관한 무수한 임상 시험 논문들이 발표되고 있고, 특히 무작위 대조 연구가 증가하고 있다. 무작위 대조연구는 비뚤림(bias)을 피 할 수 있기 때문에 치료적 중재의 효과를 평가하기 위한 가장 믿을 만한 방법으로 고려된다. 하지만 잘못 시행된 무작위 대 조연구는 오히려 잘못된 결과를 가져올 수 있으므로, 발표된 논문 결과를 환자에게 바로 적용하기 전에, 연구의 질 평가가
}

Received: August 25, 2006, Accepted: July 14, 2009

${ }^{*}$ Corresponding Author: Soo Young Kim

Tel: 02-2224-2406, Fax: 02-2224-2409

E-mail: hallymfm@gmail.com

Korean Journal of Family Medicine

Copyrights (c) 2009 by The Korean Academy of Family Medicine 
선행되어야 할 것이다.

임상 연구의 질 평가에는 개별 지표(individual markers), 점 검표(checklist), 평가 기준(scales)의 3 가지 방법이 있는데, 특 히 평가 기준은 구체화된 형식으로 질의 정량적 평가가 가능 하며 적용이 쉬워 연구들 간의 비교가 용이한 장점이 있다. 이 러한 평가 기준에 포함 가능한 항목들 중에서 특히 무작위할 당(randomization), 이중 맹검(double blinding), 탈락에 대한 기 술의 3 가지 항목은 비뚤림을 줄이는 데에 직접적인 관련이 있 으며, Jadad 질 평가 기준(Jadad Quality Assessment Scale, Jadad scale)은 이러한 3 가지 항목으로 구성된 대표적 질 평가 도구이 다. ${ }^{1)}$

또한 개별 지표의 하나로는 배정은폐(allocation concealment)의 적절성 평가가 있다. 배정은폐란 순서의 생성을 의미 하는 무작위법과는 별개의 개념으로, 생성된 무작위 할당된 순서(randomized allocation sequence)를 수행하는 방법으로 설 명되며, ${ }^{2}$ 치료적 중재를 위해 환자들을 배정하는 데 있어 선 택 비뚤림를 피하기 위해선 적절한 배정은폐의 수행이 필요 하다. ${ }^{3)}$ 하지만 현재까진 적절한 은폐에 대한 국내 논문 현황을 관찰한 논문은 없었다.

최근 국내 무작위임상시험논문의 질 평가에 대한 관심이 높아지고 있어, 임의로 선정한 국내 5 개 주요 학회지를 대상으 로 1980년도부터 2000년도까지 발행된 주요 학회지 중 자료수 집이 가능했던 12,760 편의 원저를 분석한 이전의 연구 ${ }^{4}$ 결과, 무작위임상시험논문이 1980년대 총 30편에서 1990년대 총 74 편으로 2 배 이상 증가한 양적 성장과, 이 중 높은 질로 평가된 논문이 1980년대 2편에서 1990년대 14편으로 증가한 질적 성 장이보고되었다.

하지만 이 논문에선 Jadad 항목별 분석은 시행되지 않았고, Jadad scale만을 이용하여 질 평가를 하였으며, 질적 수준에 영 향을 미치는 관련 요인들에 대한 고찰이 없었다.

이러한 점들을 보강하기 위해, 본 연구는 대한가정의학회 지에 실린 무작위대조연구논문을 대상으로, Jadad scale을 이용 한 질 평가뿐만 아니라 개별지표인 배정은폐의 적절성도 평 가하여, 설계와 수행 모두에 있어서의 질적 성장을 관찰하는 한편, 질 평가 항목 중 특히 점수가 낮은 부분을 살펴 보고, 평 가 결과에 영향을 미칠 수 있는 인자에 대하여 고찰함으로써, 향후 대한가정의학회지의 연구 방향 제시에 도움이 되고자 한다.
방법

\section{1. 연구 대상}

1980년도 11월(제1권) 창간호부터 2005년 12월(제26권) 까지 26 년 동안 발행된 대한가정의학회지에 실린 1,290 편의 원저를 수작업으로 검색하였다.

\section{2. 무작위대조연구의 추출}

연구 방법에 ‘무작위’ 라는 용어를 사용한 전향적 실험 연 구를 가정의학과 전공자 2 인이 독립적으로 추출하였고, 추출 결과차이는 제 3 자의 조정을 통해 합의하여 결정하였다.

\section{3. 추출된 무작위대조연구 논문의 질적 평가}

Jadad scale을 이용한 질 평가와, 개별 지표로 배정은폐의 적 절성을 평가하였다. 가정의학과 전공의 2 인이 독립적으로 평 가하여 결과를 비교하였고, 결과차이는 제 3 자의 조정을 통해 합의하여 결정하였다.

\section{1) Jadad scale ${ }^{\text {1) }}$}

Jadad scale은 무작위배정 순서의 생성과 이중 맹검, 탈락에 대한 3 가지 항목을 평가한다. 총점의 범위는 0-5점이며, 총점 이3점 이상일 경우 높은 질로 평가하며, 2점 이하일 경우 낮은 질로 평가한다. 이중 맹검이 불가능한 연구의 경우 총점이 2 점 이상일 때 높은 질로 평가한다. ${ }^{5)}$

무작위할당 순서의 생성과 이중 맹검에 대해, 방법에 대한 설명 없이 언급만 되어 있는 경우 각각 1 점씩 부여하고, 적절 한 방법이 기술된 경우 각각 1 점을 더한다. 반면에 기술된 방 법이 부적절한 경우 오히려 각각 1 점을 감한다. 무작위할당 순 서의 생성 방법에 대한 적절한 기술로는 "난수표", “컴퓨터로 생성된” 등이 있고, 부적절한 기술로는 “교대 할당”, “병록 번 호나 생일을 이용한" 등이 있다. 탈락에 대한 기술로는 각 대상 군별로 탈락한 수와 이유를 명시하였을 때 1 점을 부여하며, 탈 락이 한 명도 없는 경우에도 이를 본문에 기술하여야 한다. 가 산점이나감점은 없다.

2) 배정은폐

적절한(adequate) 은폐 방법이기 위해서는 "순차적 번호가 매겨진, 불투명한, 봉합된 봉투”, “순차적 번호가 매겨진 용기”, “중앙 통제된 무작위할당” 등과 같은 서술이 있어야 한다." 
Woobin Chung, et al: Quality Assessment of Randomized Controlled Trials in the Journal of the Korean Academy of Family Medicine

Table 1. Characteristics of randomized controlled tiral according to publication years.

\begin{tabular}{lccccc}
\hline Year & Original articles & $\begin{array}{c}\text { Randomized } \\
\text { controlled trials (\%) }\end{array}$ & Jadad scale mean & High quality* (\%) & $\begin{array}{c}\text { Adequate allocation } \\
\text { concealment (\%) }\end{array}$ \\
\hline $1980-1989$ & 92 & $1(1.1)$ & 1.0 & $0(0.0)$ & $0(0)$ \\
$1990-1999$ & 742 & $10(1.4)$ & 1.6 & $4(40.0)$ & $0(0)$ \\
$2000-2005$ & 456 & $12(2.6)$ & 2.17 & $4(33.3)$ & $2(16.7)$ \\
\hline Total & 1,290 & $23(1.8)$ & 1.87 & $8(34.8)$ & $2(8.7)$ \\
\hline
\end{tabular}

*Jadad scale score 3 or more, if double blinding was impractical 2 or more.

Table 2. Domain-specific adequacy of publicated trials as assessed by Jadad scale.

\begin{tabular}{lc}
\hline \multicolumn{1}{c}{ Domains } & No. of studies rated as adequate (\%) \\
\hline Randomization & $8(34.8)$ \\
Double-blinding & $2(13.3)$ \\
Reporting of withdrawals & $10(43.5)$ \\
\hline
\end{tabular}

Excluded 8 studies that double-blinding was impractical or unethical.

\section{4. 질적 평가 결과의 제시}

$\mathrm{Jadad}$ 총점의 평균과 높은 질의 비율, 적절한 은폐를 수행 한 비율에 대한 연도별 평가는, 추출된 무작위대조연구 논문 의 수가 적기 때문에, 10 년 단위로 그룹화 하여 평가하였다. Jadad scale의 항목별 분석 중 이중 맹검에 대한 항목은 이중 맹 검이 불가능한 연구를 제외하고 분석하였다. 이중 맹검이 불 가능한 경우 총점이 3 점이므로 이 경우 5점 만점으로 환산하 여 처리하였다.

\section{5. 무작위대조연구 논문의 질적 수준과 관련 요인} 논문의 질적 수준과 관련 있는 요인들로 첫째, 중재가 약 품 투여, 둘째, 특정 기관의 연구비 또는 중재가 되는 제품 후 원, 셋째, 임상시험 윤리위원회의 심의 통과 유무를 조사하였 다.

\section{결과}

\section{1. 무작위대조연구논문의 연도에 따른 양적 변화} (Table 1)

대한가정의학회지에 실린 무작위대조연구논문은 총 23 편으로, 1980 년대에 원저의 $1.09 \%$ (1편)에 불과했던 것이
1990 년대에는 10 편(1.35\%), 2000년에서 2005년까지는 12편 $(2.63 \%)$ 으로 증가하였다.

\section{2. 무작위대조연구논문의 연도에 따른 질적 변화} (Table 1)

1) Jadad Quality Assessment Scale

1980 년대의 경우 Jadad scale 평균은 1 점에 불과하였으나 1990년대엔 1.6점, 2000년에서 2005년까지는 2.17점으로 미약 하지만 증가 추세를 보였다. 높은 질로 평가된 논문은 1980년 대 0편(0.00\%)에서 1990년대 4편(40.00\%), 2000년에서 2005년 까지 4편(33.33\%)으로 1990년대 증가한 이후 유사한 양상을 보였다.

\section{2) 배정은폐}

무작위대조 연구 논문 중에서 적절한 은폐 방법을 보인 논 문은 2편(8.70\%)에 불과하였고, 부적절한 은폐는 3편(13.04\%) 이었다. 은폐 방법에 대해 언급하지 않은(unclear) 논문이 18편 (78.26\%)으로 대부분을 차지하였다. 연도별로는 1980년대와 1990년대에는 적절한 은폐 방법을 보인 논문이 한편도 없었 으나, 2000년대에 들어서 2편(16.67\%)에서 적절한 은폐 방법 이 관찰되었다.

\section{Jadad Quality Assessment Scale의 항목별 분 석(Table 2)}

총 23편을 대상으로 Jadad scale의 3가지 항목 각각에 대해 분석한 결과, 탈락에 대해 항목에서 가장 높은 비율로 기술되 었고(43.48\%), 적절한 무작위 할당이 $34.78 \%$, 적절한 이중 맹 검의 수행은 $13.33 \%$ 에서 관찰되었다. 질 평가 총점이 낮은 주 요 원인은 적절한 이중 맹검이 수행 되지 않은 것이다. 이중 맹 검이 불가능했던 논문은 총 8 편(34.78\%)으로, 치료적 중재가 운동 요법, 환자 교육, 전화상담, 대장전처치, 새로운 화상치료 
Table 3. Factors associated with randomized controlled trial quality.

\begin{tabular}{llcccc}
\hline & Factors & RCT (\%) & Jadad scale mean & High quality* (\%) & $\begin{array}{c}\text { Adequate allocation } \\
\text { concealment (\%) }\end{array}$ \\
\cline { 2 - 6 } Type & Drugs & $10(43.5)$ & 2.1 & $3(30.0)$ & $2(20)$ \\
& Non-drug & $13(56.5)$ & 1.69 & $5(38.5)$ & $0(0)$ \\
Funding source & Yes & $4(17.4)$ & 2.5 & $2(50.0)$ & $2(50)$ \\
& No & $19(82.6)$ & 1.74 & $6(31.6)$ & $0(0)$ \\
Review of IRB & Yes & $2(8.7)$ & 4 & $2(100.0)$ & $2(100)$ \\
& No & $21(91.3)$ & 1.86 & $6(28.6)$ & $0(0)$ \\
\hline
\end{tabular}

RCT: randomized controlled trial, IRB: Institutional Review Board.

*Jadad scale score 3 or more, if double blinding was impractical 2 or more.

Table 4. Factors associated with randomized controlled trial quality according to Jadad scale domain.

\begin{tabular}{llccc}
\hline \multicolumn{1}{c}{ Factors } & & Adequate randomization (\%) & Adequate double blinding* (\%) & Description of withdrawals (\%) \\
\hline Type & Drugs & $2(20.0)$ & $2(22.2)$ & $4(40.0)$ \\
& Non-drug & $6(46.2)$ & $0(0.0)$ & $6(46.2)$ \\
Funding source & Yes & $2(50.0)$ & $1(25.0)$ & $2(50.0)$ \\
& No & $6(31.6)$ & $1(9.1)$ & $8(42.1)$ \\
Review of IRB & Yes & $2(100.0)$ & $1(50.0)$ & $1(50.0)$ \\
& No & $6(28.6)$ & $1(7.7)$ & $9(42.9)$ \\
\hline
\end{tabular}

IRB: Institutional Review Board.

*Excluded 8 studies that double-blinding was impractical or unethical.

법이었고, 이중 맹검 항목의 분석은 이러한 8 편의 논문을 제외 한 후시행하였다.

\section{4. 질적 수준과 관련 요인들의 분석(Table 3)}

중재가 약품 투여인 경우와 후원을 받은 경우, 임상시험 윤리 위원회 심의를 받고 통과한 경우의 논문이 Jadad scale 평 균이 높았으며, 높은 질로 평가받은 비율은 임상시험 윤리 위 원회 심의를 통과한 경우와 후원을 받은 경우에서 높았으며 중재가 약품인 경우는 낮았다. 한편 적절한 은닉의 수행은 각 각의 관련 요인이 있는 경우에서만 관찰되었다.

\section{5. 질적 수준 관련 요인들의 Jadad scale 항목 별 분석(Table 4)}

중재가 약품 투여인 논문들은 이외의 논문들보다 이중 맹 검이 가능한 논문의 비율과 적절한 이중 맹검의 비율이 높았 으나, 적절한 무작위 할당 비율은 더 낮았다. 특정 기관의 후원 을 받거나 임상시험 윤리위원회 심의를 통과한 논문들은 모
든 항목에서 우월하였다.

고찰

본 논문의 연구 결과, 국내 가정의학회지 창간호 이후부터 2005년까지의 무작위임상시험논문들은 미약하나마 꾸준히 양적 및 질적 성장을 하고 있어서, 1980년대에 비해 2000년대 에 들어서는 무작위임상시험논문의 수가 2 배 이상 증가하였 고, Jadad scale 평균 역시 2배 이상 상승하였으며, 높은 질로 평 가 받은 논문의 수도 1980년대 0편에서, 1990년대 4편, 2000년 대 들어서는 4편으로 증가하였다. 적절한 은닉 방법은 2000년 대에 들어서야 단 2 편(8.70\%)에서 관찰되었다. 또한 총점을 낮 추는 가장 큰 원인은 적절한 이중 맹검의 부족이었다.

본 연구는 대한가정의학회지를 포함한 국내 5 개 학회지를 분석한 이전의 연구 ${ }^{3}$ 와는 몇 가지 차이점이 있었다. 우선 이전 의 논문에선 1980 년부터 2000 년까지 Jadad scale 총점이 4점 이 
상인 논문은 없었으나, 동일 기간에 본 논문에서는 2 편의 논문 이 관찰되었다. 이러한 차이는 이전의 연구가 자료 수집이 가 능했던 원저만을 대상으로 했음에 비하여, 본 연구는 수작업 으로 빠짐없이 검색한 모든 원저를 대상으로 했기 때문이라 생각한다. 또한 높은 질로 평가된 논문의 수에서도 차이를 보 이는데, 이는 이 중 맹검이 불가능한 연구에 대해서 총점 2점 이상으로 평가 기준을 조정한 본 연구와는 달리 이전의 연구 에서는 총점 3점 이상의 기준을 그대로 적용하였기 때문이라 생각한다.

더불어 본 연구는 무작위임상시험논문의 추출과 질 평가 모두에 있어서, 일정 기간 동안 근거 중심 의학 교육을 받은 가 정 의학 전공의 2 인의 개별적 이중 평가뿐만 아니라, 평가에 차이가 있을 때에는 제 3 자인 가정 의학 전문의 1 인이 조정 과 정을 수행하였으므로, 객관성과 신뢰성이 좀 더 높을 것이다.

한편 본 논문은 질적 평가의 개별 지표로 은폐 방법의 적 절성도 평가하였다. 무작위 순서가 잘 생성되었다고 하더라 도 적절한 은폐 방법 없이는 수행하는 과정에서 계획된 무작 위할당이 훼손되는 경향이 있었으며,4) 그런 이유로, 외국의 경우 적절한 은폐 방법은 질 평가의 중요한 부분으로 여기고 있다. 실제로 부적절한 배정 은폐 방법은 치료 효과를 실제보 다 과장되게 평가할 가능성이 있다. 실제로 외국의 몇몇 연구 ${ }^{6.8)}$ 에서 부적절하거나 명확하지 않은 은닉을 수행한 연구는 중 재의 효과를 $40 \%$ 이상 과장할 수 있다는 결과가 나오기도 하 였다. 외국의 경우 2000 년 1 월부터 12 월까지 발간된 4 개 주요 학술지(BMJ,JAMA, Lancet, New England Journal of Medicine)를 분석한 결과 은폐 방법이 부적절하거나 불확실한 비율이 $46 \%$ 였으며, ${ }^{9)}$ 국내의 현황은 아직 보고된 바 없다.

본 논문을 통해서, 향후 대한가정의학회의 연구자들이 이 중 맹검과 은폐 방법에 대한 올바른 이해와 적절한 수행에 세 심한 주의를 기울인다면, 관찰된 질적 성장 추세를 더욱 가속 화시킬수있음을 알수 있었다.

마지막으로, 중재의 종류가 약품 투여인 경우, 특정 기관 의 후원, 임상시험 윤리위원회의 심의 통과의 3 가지 요인은 각 각 무작위대조연구의 질적 수준과 관련이 있었다. 중재가 약 제인 경우는 위약을 사용한 이중 맹검의 수행이 좀 더 수월했 을 것이며, 이중 맹검이 가능한 논문의 수도 많아지기 때문에, 적절한 이중 맹검의 비율이 높아졌을 가능성이 있다. 하지만 적절한 무작위 할당, 탈락의 기술 등은 약제 투여 무작위 대조 연구에서 높지 않았는데 그러한 결과가 나온 이유는 불확실 하다.

특정 기관의 후원이 있는 경우는 적절한 이 중 맹검의 비 율이 높을 뿐만 아니라 적절한 무작위할당의 비율도 높은 것
이 질적 상승과 연관을 보였다. 이는 재정적 지원을 받아 좀 더 규모 있는 연구 설계가 가능하기 때문이라고 생각되며, 일차 진료에서의 치료 효과 검증 필요가 증가하는 현실에서 좀 더 많고 적극적인 후원이 이루어지는 환경이 필요하다.

또한 임상시험 윤리위원회의 심의를 거친 논문 2 편은 대 부분 항목에서 질이 높았다. 임상시험 윤리위원회의 심의를 거친 논문은 연구 계획 단계에서 설계와 수행의 타당성을 인 정받았다는 의의를 가진다. 특히 무작위 대조 연구의 경우는 임상시험 윤리위원회의 심의가 국제적 표준이라고 할 수 있 지만 국내에서는 과거 이러한 윤리 규정에 대한 인식이 강하 지 못하였다. 현재 국내 대부분 수련기관에서 임상시험 윤리 위원회를 운영하고 있거나 신설하려는 움직임을 보이고 있어 서 국내 연구의 질적 향상에 도움을 줄 것으로 예상할 수 있다.

\section{요약}

연구배경: 최근의 국내 연구에선 무작위대조시험의 양적 증 가가 관찰되지만, 질이 낮은 무작위대조연구는 오히려 잘못 된 결과를 초래하므로 논문의 질 평가가 요구된다. 본 연구는 대한가정의학회지를 대상으로 질 평가를 시행하여 양적 성장 뿐만 아니라 질적 성장도 확인하고자 한다.

방법: 1980년도부터 2005년도까지 발행된 대한가정의학회 지의 원저 1,290 편을 대상으로 무작위대조연구논문을 추출한 후, Jadad Quality Assessment Scale (Jadad scale)을 이용한 질 평가 와 배정 은폐 방법의 적절성 평가를 시행하였다. 논문의 추출 과 평가는 2 인의 연구자가 독립적으로 시행하였고 이견은 제 3자가 조정하였다. 또한 질적 수준에 영향을 미칠 수 있는 요 인들을 분석하였다.

결과: 추출된 무작위대조시험논문은 총 23 편으로 1,980 년대 원저의 $1.09 \%$ 에서 2000 년대 $2.63 \%$ 로 증가하였다.Jadad scale 총 점 평균은 1980 년대 1점에서 2000년대 2.17점으로 증가하였 고, 높은 질로 평가받은 논문도 $0 \%$ 에서 $33.33 \%$ 로 증가하였지 만, 적절한 은닉 방법은 2000년대 들어서야 단 2편에서 관찰되 었다. 질 평가 항목 중 가장 부족한 항목은 적절한 이중 맹검이 었고, 중재가 약품이거나 특정 기관의 후원을 받은 논문 또는 임상시험 윤리위원회 심의를 받은 논문들은 그 외의 논문들 보다 높은 질적 평가를 받았다.

결론: 대한가정의학회지에 실린 무작위 대조시험 논문은 1980년도 창간호부터 2005년도까지 꾸준한 양적 성장과 질적 성장을 하였지만 아직 충분치 않으므로, 향후 연구자들은 특 히 적절한 이중 맹검과 은닉 방법이 시행될 수 있도록 노력이 
필요하겠다. 또한 연구의 질적 향상을 위해 좀 더 많은 후원을 받을 수 있는 환경이 조성되어야 하고, 임상시험 윤리위원회 심의 통과에도 관심을 기울여야 할 것이다.

중심단어: 대한가정의학회지; 무작위대조시험; 질적 평가; 은닉 방법

\section{REFERENCES}

1. Jadad AR, Moore RA, Carroll D, Jenkinson C, Reynolds DJ, Gavaghan DJ, et al. Assessing the quality of reports of randomized clinical trials: is blinding necessary? Control Clin Trials 1996;17:1-12.

2. Moher D, Cook DJ, Jadad AR, Tugwell P, Moher M, Jones A, et al. Assessing the quality of reports of randomised trials: implications for the conduct of meta-analyses. Health Technol Assess 1999;3:i-iv, 1-98.

3. Kim SW, Choi YS, Ahn HS, Lee HY, Ahn DS, Lee YM. Quantity and quality assessment of randomized controlled trials published in five Korean medical journals, from 1980 to 2000. J Korean Acad Fam Med 2004;25:118-25.
4. Schulz KF, Grimes DA. Allocation concealment in randomised trials: defending against deciphering. Lancet 2002;359:614-8.

5. Weiger WA, Smith M, Boon H, Richardson MA, Kaptchuk TJ, Eisenberg DM. Advising patients who seek complementary and alternative medical therapies for cancer. Ann Intern Med 2002;137:889-903.

6. Schulz KF, Chalmers I, Hayes RJ, Altman DG. Empirical evidence of bias. Dimensions of methodological quality associated with estimates of treatment effects in controlled trials. JAMA 1995;273:408-12.

7. Moher D, Pham B, Jones A, Cook DJ, Jadad AR, Moher M, et al. Does quality of reports of randomised trials affect estimates of intervention efficacy reported in meta-analyses? Lancet 1998;352:609-13.

8. Jüni P, Altman DG, Egger M. Systematic reviews in health care. Assessing the quality of controlled clinical trials. BMJ 2001;323:42-6.

9. Hewitt C, Hahn S, Torgerson DJ, Watson J, Bland JM. Adequacy and reporting of allocation concealment: review of recent trials published in four general medical journals. BMJ 2005;330:10578. 